\title{
Frontoinsular cortical microstructure is linked to life satisfaction in young adulthood
}

\author{
Ryan P. Cabeen ${ }^{1} \cdot$ Arthur W. Toga ${ }^{1} \cdot$ John M. Allman ${ }^{2}$
}

Received: / Accepted:

\begin{abstract}
Life satisfaction is a component of subjective wellbeing that reflects a global judgement of the quality of life according to an individual's own needs and expectations. As a psychological construct, it has attracted attention due 5 to its relationship to mental health, resilience to stress, and other factors. Neuroimaging studies have identified neurobiological correlates of life satisfaction; however, they are limited to functional connectivity and gray matter morphometry. We explored features of gray matter microstructure ob10 tained through compartmental modeling of multi-shell diffusion MRI data, and we examined cortical microstructure in ${ }^{35}$ frontoinsular cortex in a cohort of 807 typical young adults scanned as part of the Human Connectome Project. Our experiments identified the orientation dispersion index (ODI), 15 and analogously fractional anisotropy (FA), of frontoinsular cortex as a robust set of anatomically-specific lateralized diffusion MRI microstructure features that are linked to life satisfaction, independent of other demographic, socioeconomic, and behavioral factors. We further validated

20 our findings in a secondary test-retest dataset and found high reliability of our imaging metrics and reproducibility of outcomes. In our analysis of twin and non-twin siblings, we found basic microstructure in frontoinsular cortex to be strongly genetically determined. We also found a more moderate but
\end{abstract}

ture as it relates to life satisfaction in frontoinsular cortex. Our findings suggest a potential linkage between well-being and microscopic features of frontoinsular cortex, which may reflect cellular morphology and architecture and may more broadly implicate the integrity of the homeostatic processing performed by frontoinsular cortex as an important component of an individual's judgements of life satisfaction.

Keywords subjective well-being - diffusion microstructure $\cdot$ frontoinsular cortex $\cdot$ young adulthood $\cdot$ von Economo neurons

\section{Introduction}

Subjective well-being is a concept that encompasses the many facets of an individual's psychological state concerning whether life is desirable, pleasant, and good (Diener, 2009). As a topic of research, subjective well-being has been approached theoretically to differentiate it from happiness (Shin and Johrson, 1978) and to subdivide it into hedonic and eudaimonic components (Ryan and Deci, 2001; Ryff et al., 2004). Life satisfaction is an element of subjective well-being that is a judgement of the global quality of life, as assessed by an individual's own chosen criteria regarding their needs and expectations (Shin and Johnson, 1978). Viewed as a psychological construct, life satisfaction has raised substantial interest, as it reflects personal orientations such as meaning, pleasure, and engagement (Peterson et al., 2005), and it is a factor in mental health and response to stress, anxiety, and depression (Mahmoud et al. 2012, Pavot and Diener 2009); furthermore, it may provide a beneficial target for guiding public policy beyond economic measures 55 of wealth (Diener and Seligman, 2004). Several multi-item questionnaires have been developed to provide a life satisfaction measurement scale representing "a cognitive and global evaluation of the quality of one's life as a whole"
${ }^{2}$ Division of Biology and Biological Engineering
California Institute of Technology

Pasadena, CA, USA
Corresponding Author: Ryan P. Cabeen

${ }^{1}$ Laboratory of Neuro Imaging

USC Stevens Neuroimaging and Informatics Institute

Keck School of Medicine of USC

University of Southern California

Los Angeles, CA, USA 
(Pavot and Diener, 2009), and these scales have been widely 60 applied and shown to be highly reliable (Diener et al., 1985; Samman, 2007; Salsman et al., 2014, 2013). The genetic heritability of life satisfaction has also been measured in 10 separate studies in monozygotic twins (total $\mathrm{N}=44,450$ ). $\mathrm{A}_{11}$ meta-analysis of these studies found an average heritability index of 0.32 with a $95 \%$ confidence range between 0.29 and 0.35 (Bartels, 2015).

An emerging goal of well-being research is to under-120 stand its underlying neural substrate, and in particular, to characterize how brain structure and function relates to the

70 psychological construct of life satisfaction. Magnetic resonance imaging (MRI) has been the primary tool for investigating such brain factors related to subjective well-being, ${ }^{125}$ with studies looking at both functional connectivity and gray matter morphometry (Machado and Cantilino, 2017). Func-

75 tional MRI studies have employed resting-state approaches to identify low frequency oscillatory features associated with well-being, including effects in temporal, insular, and sub-130 cortical regions (Kong et al., 2015b c). More targeted seedbased functional MRI has shown associations between insu-

80 lar connectivity and subjective well-being (Li et al., 2020). Furthermore, functional MRI work has looked more specifically at life satisfaction and mediation effects, finding the ${ }^{135}$ insula and dorsal anterior cingulate cortices to be activated with mediation by resilience (Kong et al. 2015d), insular 5 and dorsolateral prefrontal cortex connectivity conditional on a valenced face-word task (Kim et al. 2016), and activation of ventrolateral prefrontal and posterior cingulate ${ }^{140}$ cortices mediated by self-respect and self-criticism (Kyeong et al. 2020). The meta-analysis by Tanzer and Weyandt (2019)

90 provides a quantitative summary of functional MRI findings, which include meaningful activation clusters identified in the claustrum, insula, basal ganglia, and thalamus. In par-145 ticular, in our review of Tanzer and Weyandt (2019), we found that functional activity related to happiness based on 5 meaning, which is closest to eudiamonia, was localized to frontoinsular cortex in both the right and left hemispheres, as based on a comparison of their reported coordinates and 150 our own manually delineated frontoinsular region. Beyond functional imaging, structural MRI studies have employed voxel-based morphometry to estimate local changes in gray matter volume, finding insular gray matter volume related to eudaimonia (Lewis et al., 2014), differences with subjective $e_{55}$ well-being in the precunus (Sato et al. 2015), and a relationship between measures of quality-of-life and anterior insula 105 volume in older adults Ourry et al.(2021). Some works have further identified hippocampal volumetric differences with subjective well-being (Van't Ent et al., 2017), and a com-160 bination of subcortical and cortical changes to be mediated by self-esteem (Kong et al., 2015a). Cortical thickness has ciated with life satisfaction with mediation by relationship status (Zhu et al., 2018).

Together, these findings show widespread associations between well-being scales and brain structure and function, with some convergence in frontal, insular, and posterior cingulate areas; however, they do not tell the whole story, as structural imaging metrics derived from voxel-based morphometry and cortical thickness analysis are somewhat nonspecific to underlying cellular changes. Diffusion MRI is a well-established technique that has yet to be applied to study subjective well-being, and it offers a unique probe into the microstructural features of brain tissue, which may reflect changes in cellular morphology and organization (Alexander et al., 2019). While typically applied in white matter imaging, computational modeling techniques are making it possible to characterize the microstructure of the cortex $[\mathrm{Fuk} \mu-$ tomi et al., 2018). Such analyses have previously proven challenging due to limited image resolution, geometric distortion, and partial volume effects of diffusion MRI scans. However, these issues are more recently mitigated by higher resolution imaging sequences, multi-shell gradient protocols for separating tissue compartments, and by sophisticated artifact correction tools to reduce susceptibility-induced geometric distortion. This has led the way to work showing how cortical microstructure reflects aspects of environmental exposure, genetics, and neurodegeneration (Genç et al. 2018; Schmitz et al., 2019, Cabeen et al., 2020, Baxi et al. 2020 Torso et al.; Caron et al., 2020). In contrast to white matter imaging, microstructure modeling of gray matter may potentially reveal aggregate features of soma density, myelination, dendritic complexity, and laminar architectural features of cortex (Nazeri et al., 2020). This provides a valuable direction for investigating well-being and understanding how it relates to the integrity of cortical homeostatic processes beyond what is available through volumetric analysis.

Our present study investigates how such cortical microstructural features are related to life satisfaction, as a part of ongoing research characterizing features of frontoinsular cortex and other subcortical brain regions related to decision making and emotion regulation. Our motivation for focusing on frontoinsular cortex is due to its central role in homeostatic processing (Craig, 2009), emotional awareness $(\mathrm{Gu}$ et al. 2013), salience processing (Seeley et al. 2007), and its distinct cytoarchitectonic features (Allman et al., 2010). We focused on neurite orientation dispersion and density imaging (NODDI), as its component measures depict distinct cytoarchitectural features, i.e. separating features of cellular density and myelination from dendritic complexity and cell morphology (Fukutomi et al. 2018). After identifying a relationship with life satisfaction in frontoinsular cortex, we broadened our analysis to assess lateralization, the anatomical specificity of our findings across the cortex, and the relationship of frontoinsular microstructure to other demo- 
graphic and behavioral variables. We further expanded our 210

165 analysis with a validation experiment conducted with a secondary test-retest dataset to establish the reliability of our microstructural metrics and to examine replicability of our specific outcomes across cohorts and scanning sessions. We further examined a cohort of twin and non-twin siblings to215 determine the heritability of frontoinsular microstructure parameters and to examine inter-sibling differences in microstructure and life satisfaction. Our data was obtained from the Human Connectome Project, which provides a large cohort of typical young adults scanned with a high quality diffusion

175 MRI sequence suitable for multi-modal analysis with cortical surface modeling. We next describe the design and procedures of our experiments, present our findings, and subsequently discuss our results, their interpretation and implications, and potential limitations.

\section{Participants and Datasets}

Data for our experiments were acquired from participants as part of the Human Connectome Project (HCP). We performed a multi-modal analysis that included both $\mathrm{T}_{1}$-weighted (T1wMRI) and diffusion-weighted MRI (dwMRI) data, and in total, we included 807 participants with scans that pass quality control and completed image processing. With approval from the University of Southern California Institutional Review Board, we accessed and analyzed demographic and behavioral data from the restricted data release. We collected scores from the Emotion Battery of the NIH Toolbox General Life Satisfaction Survey (Salsman et al. 2013, 2014) 1 According to the toolbox technical manual, the questionnaire is designed to measure "one's cognitive evaluation experiences and is concerned with whether people like their lives or not." We also collected other behavioral variables to determine the specificity of our effect. This included two other NIH Toolbox well-being measures: positive affect and a combined variable for meaning and purpose. From the set of instruments from the Achenbach System of Empirically Based Assessment (ASEBA) (Achenbach and Rescorla 2003), we also retained the adult selfreport thought problems scale (Abdellaoui et al., 2012). We used this scale to operationalize a summary measure of negative intrusive thinking, as the questionnaire includes selfreported measures of hallucinations, self-destructive thoughts, repetitive behavior, and other factors that negatively impact daily life. To provide general summaries of cognitive performance, the overall accuracy in working memory and the

1 https://www.healthmeasures.net/ explore-measurement-systems/nih-toolbox/ intro-to-nih-toolbox/emotion cognitive function composite score were extracted from the NIH Toolbox measures. We also retained the following demographic variables from the semi-structured assessment for the genetics of alcoholism (SSAGA) that are possibly relevant to life satisfaction: income, relationship status, employment status, level of education, and body mass index.

Image Acquisition and Preprocessing

The data were collected using the imaging protocol designed for the Human Connectome Project, which we briefly summarize here. The T1wMRI and dwMRI data from the HCP ner using a 32-channel head coil (Glasser et al., 2013; Van Essen et al. 2013). The T1wMRIs were acquired using a 3D MPRAGE sequence with $0.7 \mathrm{~mm}$ isotropic resolution (FOV $=224 \mathrm{~mm}$, matrix $=320,256$ sagittal slices in a single slab), repetition time $(\mathrm{TR})=2400 \mathrm{~ms}$, echo time $(\mathrm{TE})=2.14 \mathrm{~ms}$, inversion time $(\mathrm{TI})=1000 \mathrm{~ms}$, flip angle $=8^{\circ}$, bandwidth $=210 \mathrm{~Hz}$ per pixel, echo spacing $=7.6 \mathrm{~ms}$, and phase encoding undersampling factor GRAPPA $=2.10 \%$. dwMRIs were collected with a single-shot $2 \mathrm{D}$ spin-echo EPI acquisition with a multi-band factor of $3,1.25 \mathrm{~mm}$ isotropic voxels with FOV PE by Readout $=210$ x 180; matrix size PE by Readout $=144 \times 168 ; 111$ interleaved slices without gap; left-right and right-left phase encoding; flip angles $=78^{\circ}$ and $160^{\circ}$. For each phase encoding direction, the diffusion sampling scheme consisted of 18 baseline scans and 270 diffusion-weighted scans acquired using single diffusion encoding across 3 shells with $b=1000,2000$, and $3000 \mathrm{~s} / \mathrm{mm}^{2}$; all dwMRI scans had TE $=89 \mathrm{~ms}$ and $\mathrm{TR}=5.5 \mathrm{~s}$. Each shell included 192 data points representing 90 diffusion gradient directions and six $b=0$ shells acquired twice resulting in 270 non-collinear directions for each PE. Total acquisition time was approximately 54 min (6 segments of 9 min each). dwMRI data were preprocessed with the HCP workflow (Sotiropoulos et al., 2013). This included the sophisticated approach for correction of artifact due to motion and eddy-current and susceptibility induced geometric distortion in FSL EDDY. Using an additional set of diffusion MRI scans collected with reversed phase encoding, this scheme estimates and corrects for the off-resonance field and subject head motion using a Gaussian process framework for robust non-parametric interpolation of the dwMRI signal. This is a crucial step for mapping cortical microstructure, as surfacebased mapping of microstructure parameters requires accurate alignment between T1wMRI and dwMRI data, which can be otherwise disturbed by susceptibility-induced geometric distortion. 

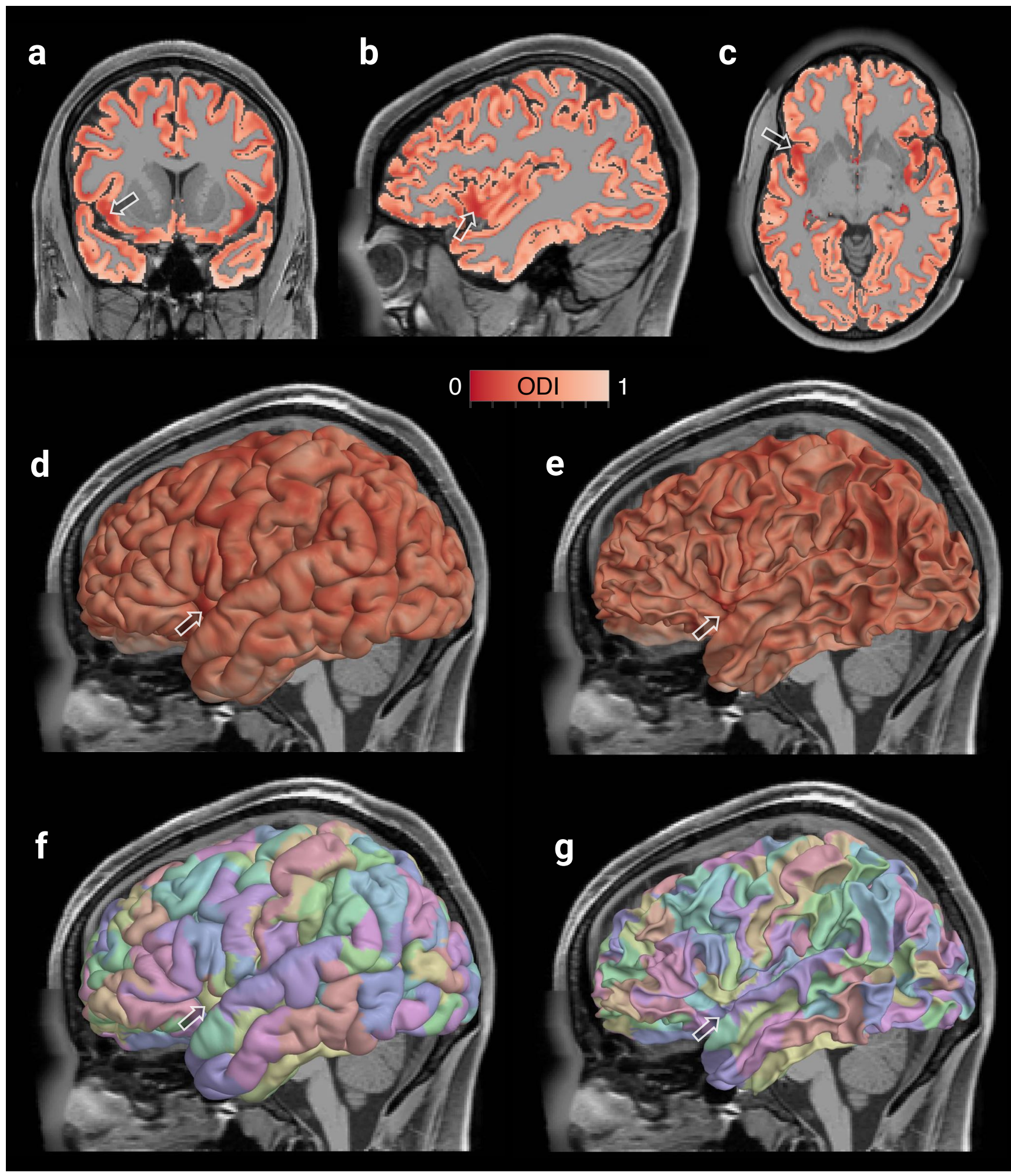

Fig. 1 An overview of the imaging results illustrated using data from a randomly selected individual. The top row shows an overlay of the orientation dispersion index on the structural T1 image in coronal (a), sagittal (b), and axial (c) planes. Frontoinsular cortex is identified by an arrow, which lies in an area with lower dispersion than other parts of cortex. The middle row shows an overlay of orientation dispersion on a cortical surface models, which are produced using a statistical mapping procedure that samples microstructure parameters between the pial (d) and white matter (e) boundary surfaces. The bottom row shows the HCP multi-modal parcellation used in our analysis, where the pial (f) and white matter $(\mathrm{g})$ boundary surfaces are randomly colored to distinguish separate parcels. The agranular anterior insular cortex (AAIC) parcel is identified by an arrow, which also coincides with frontoinsular cortex. 
Image Analysis

Our image analysis took a multi-modal approach that uses intra-subject registration to accurately segment cortical strucOur initial experiment performed a simple region-of-interest based approach to investigate microstructure in frontoinsu-315 lar cortex and several other regions. We subsequently expanded this analysis to the entire cortex and performed further validation experiments. Our analysis is similar to our previous work (Cabeen et al. 2020), with several algorithmic refinements in an effort to make our pipeline into a tool 320 that can be applied by other researchers. The data were analyzed using the LONI Pipeline (Dinov et al., 2009) with Cabeen et al. 2018), Freesurfer (Fischl 2012), FSL (Jenkinson et al. 2012), ANTs (Avants et al., 2008), and DTI-TK 325 (Zhang et al., 2006). The specific steps of our analysis are described in detail as follows.

The dwMRI data were denoised using a non-local means filter and microstructure parameters were obtained using two multi-shell modeling approaches. First, we performed neu- ${ }^{330}$ rite orientation dispersion and density imaging (NODDI) (Zhang et al. 2012) and estimated its parameters using a 280 non-linear fitting approach accelerated using the spherical mean technique (Cabeen et al., 2019), resulting in volumetric maps of the orientation dispersion index (ODI) and neu- ${ }_{335}$ rite density index (NDI). Because our experiments look specifically at gray matter, we used a parallel diffusivity of $1.1 \mathrm{x}$ $10^{-3} \mathrm{~mm}^{2} / \mathrm{s}$, which is an optimized value obtained from previous work (Fukutomi et al. 2018). NDI is meant to depict the proportion of neurite volume relative to the total cellular ${ }_{340}$ volume, while ODI is meant to separately depict neurite orientational heterogeneity. We also estimated diffusion tensor

290 imaging (DTI) (Basser and Jones, 2002) parameters using weighted linear least squares fitting with free-water elimination with a fixed diffusivity of $3.0 \times 10^{-3} \mathrm{~mm}^{2} / \mathrm{s}$ in the $\mathrm{s}_{345}$ isotropic compartment using the non-linear least squares appraoch of Hoy et al. (2014), resulting in volumetric maps of fractional anisotropy (FA) and mean diffusivity (MD). We used these two distinct diffusion modeling approaches to understand how they relate, because they are both widely used $_{350}$ and useful points of reference. An important piece, however, is that they both include a free-water compartment, which

300 can reduce partial volume effects due to subvoxel mixing of cerebrospinal fluid and gray matter tissue. Generally speaking, ODI and FA may reflect similar tissue properties, but ${ }_{355}$ they are inversely related, as lower neurite dispersion is reflected by an increase in tensor anisotropy.

We created a population-averaged dataset from 88 scans from the test-retest portion of the HCP dataset to serve as a template in our initial region-of-interest analysis. We used ${ }_{360}$ the tensor-based deformable registration and spatial normal- ization pipeline implemented in DTI-TK (Zhang et al., 2007)

to produce the population averaged DTI, NODDI, and T1wMRI datasets that were aligned to the IIT template (Zhang et al. 2011). Subject data from all participants was then spatially Inormalized to the template and the deformable registration maps were retained for each individual. Our inital goal was to investigate gray matter microstructure parameters in frontoinsular cortex and related structures involved with decisionmaking and emotion regulation using a region-of-interest (ROI) approach. Guided by the definition from Allman et al. (2010), we first manually delineated ROIs on coronal slices of the template for left and right frontoinsular cortex using QIT and co-registered these in subject native space and computed region-averaged parameters. The other additional structures we examined included: the nucleus accumbens, caudate, putamen, substantia nigra (separate compact and reticular parts), amygdala, hippocampus, hypothalamus, and periaqueductal gray. ROIs were obtained from the Caltech Subcortical and Amygdala Atlases (Pauli et al. 2018; Tyszka and Pauli, 2016), and the remainder were manually drawn on the population average. The Caltech atlas data were aligned to our population average T1wMRI map using ANTs diffeomorphic registration.

Given our significant findings in frontoinsular cortex, we performed a subsequent analysis investigating microstructure properties across the entire cerebral cortex, so as to determine the anatomical specificity of our results. We used a multi-modal modeling approach, in which we combined Freesurfer cortical surface models with diffusion MRI microstructure parameter maps. We processed T1wMRI data using Freesurfer version 5.3.0 to create 3D geometric models for the inner and outer cortical surface. The cortical models were aligned with dwMRI data using ANTs to computing the optimal rigid transform between the T1wMRI and average baseline diffusion image, and microstructure parameters maps were spatially normalized in the higher resolution T1wMRI space. To estimate cortical microstructure, we took a similar approach to Fukutomi et al. (2018) and used a statistical procedure to refine the alignment of the cortical surface to better match the tissue boundaries in the diffusion scan, in the event that any subtle geometric distortions remain after artifact correction. Briefly, for each subject, we computed the weighted average microstructure parameters in each vertex of the Freesurfer cortical surface, which consisted of two stages. In the first stage, the midpoint between the pial and white matter surfaces was computed and 15 sampling points were equally spaced between them. For a given microstructure parameter, the values were measured at each of the sampling point and subsequently weighted using a Gaussian function centered at the midpoint with a weight of one, with a standard deviation that gives a weighting of 0.05 at each of the inner and outer cortical boundaries. This weighting scheme is designed to 
carefully isolate gray matter voxels by reducing the influence of voxels that are closer to tissue boundaries, which are more prone to partial volume effects. In the second stage,, 415 mean and standard deviation microstructure parameters were computed and outlier values were detected and excluded using a z-score threshold of 3.0; finally, the average value was found from points that remained after outlier rejection. We then summarized the microstructure parame-420 ters in the regions defined by the HCP multi-modal parcellation (HCP-MMP-1.0), which consists of 180 cytoarchitectonically defined parcels in each hemisphere (Glasser et al. 2016). The HCP-MMP-1.0 region most closely aligned with the manually drawn frontoinsular ROI was agranular ante-425 rior insular cortex (AAIC).

\section{Statistical Analysis}

Our statistical analysis explored the relationship among behavioral and demographic variables, how they relate to cortical microstructure, and also included a validation exper-

380 iment to establish the reliability and stability of our findings, as well as tests of lateralization and heritability. We used multiple linear regression modeling to examine the relationship among variables, with the following approach. All continuous model parameters were normalized to zero-mean and unit-variance to allow their regression coefficients to be reported in standardized units. We excluded outliers using Tukey's procedure, in which high and low cutoffs were determined by 1.5 times the inter-quartile range beyond the low and high quartiles, computed using the entire cohort. We retained the $\mathrm{R}^{2}$ coefficient of determination of the model, and the statistical outcomes of each subject variable, including the standardized regression coefficient $\beta$, t-value, standard error, and p-value. The specific steps of our analyses 445 are described next.

The first step of our analysis examined demographic and behavioral variables by measuring distributional statistics and estimating linear regression models relating life satisfaction to basic demographics (age, sex, body mass index),450 other cognitive and emotional variables (memory, cognition,

400 thought problems, meaning and purpose, and positive affect), and socioeconomic factors (relationship status, income, education, and employment). We also created plots showing the relationships.

Our second step examined the relationship between life satisfaction and frontoinsular microstructure, specifically ODI and FA, separately for each hemisphere. We estimated linear regression models in a stepwise fashion to test covariates that included the other cognitive and emotional factors, 460 socioeconomic factors, and environmental factors. To determine the anatomical specificity of our findings, we then repeated this analysis across the entire cortex and created 3D visualizations showing statistical parameters for each brain area. We performed additional post-hoc statistical tests to determine the robustness of our findings to subject motion and validate our effects with permutation tests. We summarized the total motion for each participant by computing to total translational motion accumulated across the scanning session, computed by summing the magnitudes of the volume-to-volume translation from the output of FSL EDDY. For permutation testing, we used randomization of the life satisfaction scores with 10,000 samples to estimate the null distribution of the observed effect, including the newly added motion covariate. We computed the two-sided significance in a typical way by computing the proportion of samples with an absolute effect greater than our observed effect.

Our third step performed a validation experiment, which used a secondary test-retest dataset from the Human Connectome Project with two scans collected for 44 individuals. We first measured reliability of cortical microstructure parameters using the intraclass correlation (ICC) and the coefficient of variation $(\mathrm{CoV})$, with a comparison of FA and ODI metrics. Given a mean parameter value $\mu$, a within-session variance $\sigma_{\text {within }}$, and a between-subjects variance $\sigma_{\text {between }}$, the ICC was computed by $\left(\sigma_{\text {between }}-\sigma_{\text {within }}\right) /\left(\sigma_{\text {between }}+\right.$ $\left.\sigma_{\text {within }}\right)$, and the $\mathrm{CoV}$ by $\sigma_{\text {within }} / \mu$. We then repeated our life satisfaction analysis to determine the replicability of the findings within each test and retest group. We grouped left and right hemisphere variables to increase the power of the analysis, and we estimated an interaction with the timepoint variable to determine if there was a difference in effect sizes across timepoints. We also computed the correlation between test-retest pairs of life satisfaction and frontoinsular cortical microstructure parameters.

Finally, we performed laterization and heritability experiments. We compare four pairs left and right microstructure parameters, which include FA and ODI in each of frontoinsular cortex and the MMP AAIC parcel. We apply a paired ttest to assess left-right differences, and we computed the lateralization index (LI) for each parameter. The LI was found by taking the ratio of the average left-right difference over the mean value of the combined left and right values, so a positive value indicates the left hemisphere had a high parameter value than the right. We computed the heritability of each microstructure parameter in a subset of the HCP cohort that were siblings. We identified sibling pairs by matching parental identifiers and then separating based on zygosity to obtain three groups: monozygotic (identical) twins, dizygotic (fraternal) twins, and non-twin siblings. We plotted microstructural parameters for each pair of twins and computed the correlation coefficient between them to determine the degree of heritability. We also computed inter-sibling differences in life satisfaction and microstructure, and we computed their correlation to further validate our findings in this sibling cohort. 


\section{Demographic and Behavioral Results}

Participants were 28.7 years old on average $(\sigma=3.67$ years $)$ across 454 female and 355 male participants, with an average life satisfaction score of $54.36(\sigma=9.39)$, and normal satisfaction showed no statistically significant difference between sexes $(p=0.786)$, nor with body mass index $(p=$ $0.094)$, but with a small decrease with age $(\beta=-0.142, p=$ $0.001)$. All considered socio-economic factors were asso$(\beta=0.127, p<0.0001)$, relationship status $(\beta=0.465, p<$ $0.0001)$, education level $(\beta=0.055, p=0.006)$, and employment status $(\beta=-0.138, p=0.004)$. Other measures of well-being were associated as well: meaning and purpose ${ }_{490}(\beta=0.044, p<0.0001)$, positive affect $(\beta=0.046, p<$ $0.0001)$, thought problems $(\beta=-0.111, p=0.0001)$, and ${ }^{535}$ cognitive performance $(\beta=0.134, p<0.0001)$. Relationship status was split roughly equally (344 true, 435 false), education level was relatively concentrated at 16 years of education, income was fairly evenly distributed, and most were employed in full time jobs $(\mathrm{N}=520)$ and fewer were part-540 time $(\mathrm{N}=139)$. Distributional summaries of demographic and behavioral data are presented in the supplemental material.

\section{Cortical Microstructure Results}

Regarding the results from our initial manually drawn regionof-interest approach, we found that frontoinsular cortical orientation dispersion was significantly associated with life satisfaction in both hemispheres (left $\beta=-0.128, p=0.0003$;550 right $\beta=-0.142, p=0.00004)$, accounting for sex, body ass index, and intracranial volume (Figure 2). Sex had a significant effect as a covariate in both hemispheres (left $\beta=$ $0.488, p<0.00001$; right $\beta=0.635, p<0.0001)$. Among other cognitive and emotional factors, the only significant 555 covariates were thought problems (left $\beta=0.111, p=0.003$; right $\beta=0.096, p=0.009$ ) and meaning and purpose (right $\beta=0.013, p=0.009$ ), while no socioeconomic variables showed an effect as covariate. Among environmental factors, THC exposure (left $\beta=0.253, p=0.031$; right $\beta=$ $0.498, p=0.00002$ ) and paternal alcohol exposure (right $\beta=0.273, p=0.006$ ) were significant covariates. No other manually-drawn regions of interest besides frontoinsular cortex showed a significant association with life satisfaction, and no effects were found with FICVF and MD. Distributional summaries and expanded results are presented in the supplemental material.

Our expanded analysis further looked at surface-based measures of cortical microstructure and showed that the only significant cortical parcel was agranular anterior insular cortex (left $\beta=-0.078, p=0.026$, right $\beta=-0.143, p=0.00003$ ).

525 Other covariates showed similar results as those from frontoinsular cortex, and when examining FA, the results showed similar associations, but with inverted effect sizes of smaller magnitude. Our post-hoc statistical effects showed that the inclusion of subject motion as a covariate did not change the magnitude or significance of our effects, nor did permutationbased estimates of significance. The results of these additional tests may be found in the supplemental material.

\section{Validation Results from Secondary Data}

Our first validation experiment investigated reliability of cortical microstructure metrics using the secondary test-retest HCP dataset (Figure 3). The distribution of ODI results across the cortex had an average ICC of $0.77(\sigma=0.10)$ and average $\mathrm{CoV}$ of $2 \%(\sigma=1 \%)$. Agranular anterior insular cortex showed a higher than average ICC (left 0.88; right 0.86) and typical $\mathrm{CoV}$ (left $2.1 \%$, right $2.4 \%$ ). The manually-drawn frontoinsular region showed a high ICC (left 0.84; right 0.88) and low $\mathrm{CoV}$ (left 1.6\%; right 1.5\%). In a direct comparison of test-retest values, we found a high correlation between scanning sessions of both life satisfaction $(\beta=0.965)$ and frontoinsular cortex (beta $=0.961)$. Our second validation experiment repeated the life satisfaction analysis for each of the test and retest cohorts, and found similar effects across the primary and secondary data $(\beta=0.220, p=0.005)$. A multiple regression model showed no significant difference in ODI between timepoints and no difference between the regression coefficients of the two models ( beta $=0.041, p=$ 0.787). Similar results were found with DTI FA, though with slightly lower ICC $(\mu=0.73, \sigma=0.13)$ and higher $\mathrm{CoV}$ $(\mu=3 \%, \sigma=1 \%)$. We found the life satisfaction score to have an ICC of 0.87 and and $\mathrm{CoV}$ of $3.6 \%$. A detailed summary of these results is provided in the supplement. 
Distribution of Frontoinsular ODI

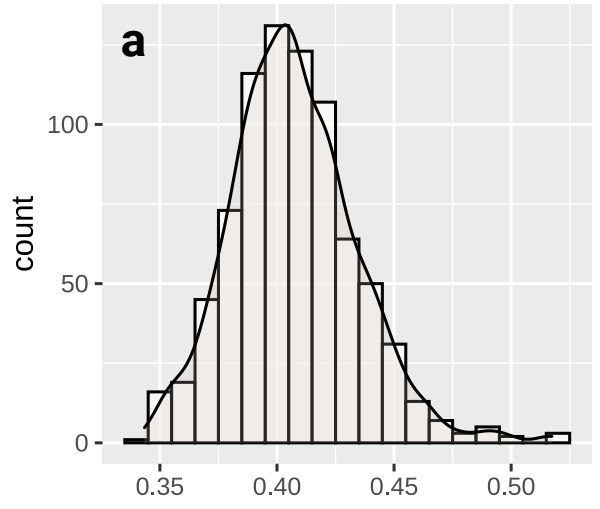

Distribution of Agranular Insular ODI

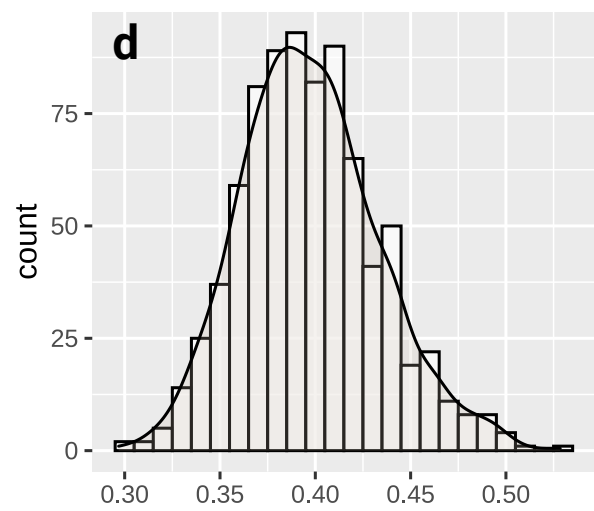

Left and Right Frontoinsular ODI vs Life Satisfaction
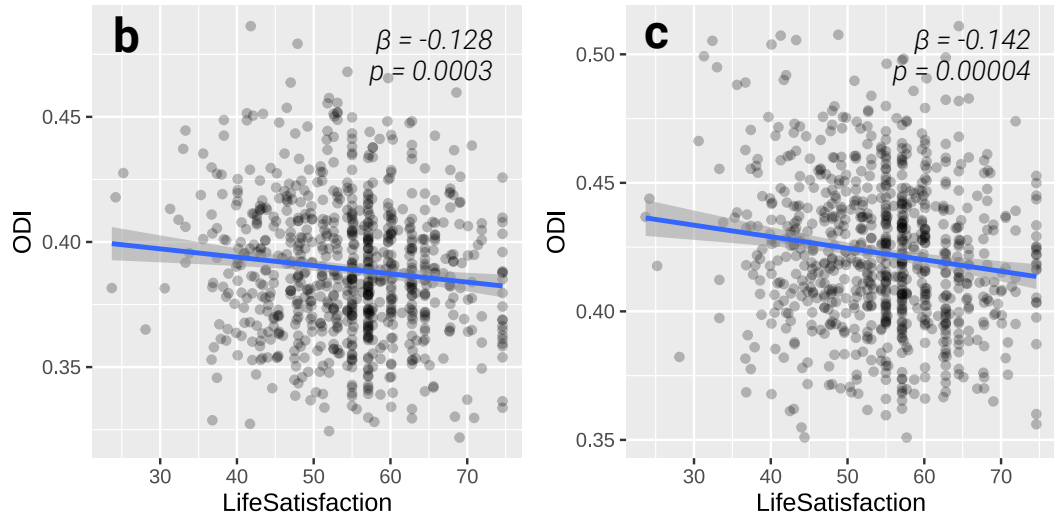

Left and Right Agranular Insular ODI vs Life Satisfaction

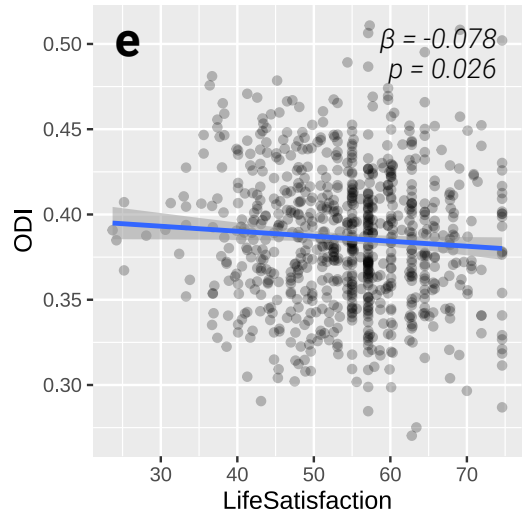

Fig. 2 Plots showing the results of our analysis comparing cortical microstructure and life satisfaction. The first panel (a) shows the overall distribution of the orientation dispersion index in frontoinsular cortex. The next two panels show the relationship between life satisfaction and frontoinsular microstructure in the left (b) and right (c) hemispheres. The first row shows results from our manually-drawn frontoinsular region, while the second row shows results from an automated surface-based approach for extracting agranular anterior insular parcel. The results show how higher life satisfaction is associated with lower dispersion, with a stronger effect in the right hemisphere. Statistical results (standardized effect size $\beta$ and p-value) were obtained from multiple linear regression modeling with demographic and behavioral covariates.

\section{Laterality and Heritability}

In our tests of laterality, we found right hemisphere ODI in ${ }^{575}$ frontoinsular cortex to be higher than the left $(\delta=-0.033, p<$ $\left.10^{-15}, L I=-0.081\right)$, and we found right hemisphere FA in frontoinsular cortex to be lower than the left $(\delta=0.013, p<$ $\left.10^{-15}, L I=0.071\right)$. Similarly, we found right hemisphere ODI in AAIC to be higher than the left $\left(\delta=-0.020, p<<^{580}\right.$ $10^{-15}, L I=-0.051$ ), and we found right hemisphere FA in AAIC to be lower than the left $\left(\delta=0.0072, p<10^{-15}, L I=\right.$ $0.045)$. Thus, ODI tended to show higher lateralization than FA, and frontoinsular cortex showed higher lateralization than AAIC.

In our tests of heritability (Figure 4), we found significant heritability in ODI in frontoinsular cortex among monozygotic twins $(\beta=0.71, p<0.0001)$, and lower heritability among non-twin siblings ( $\beta=0.244, p=0.003$ ), with in- termediate trend-level heritability in dizygotic twins $(\beta=$ $0.303, p=0.055)$. FA in frontoinsular cortex showed a similar overall trend but smaller effect sizes for all three groups: monozygotic twins $(\beta=0.644, p<0.0001)$, dizygotic twins $(\beta=0.275, p=0.19)$, and non-twin siblings $(\beta=0.194, p=$ 0.024). We found a similar pattern in AAIC results for ODI and FA, as well. We found life satisfaction to have significant heritability among all siblings with a lower effect size than microstructure ( $\beta=0.234, p=0.00002)$, but not when examining the twin subgroups of smaller sample size. In our comparison of inter-sibling differences in life satisfaction and microstructure, we found a significant association among all siblings $(\beta=-0.145, p=0.008)$, which is similar to our previous findings. A detailed summary of these results is provided in the supplement. 
Test-Retest of Life Satisfaction and Frontoinsular Microstructure

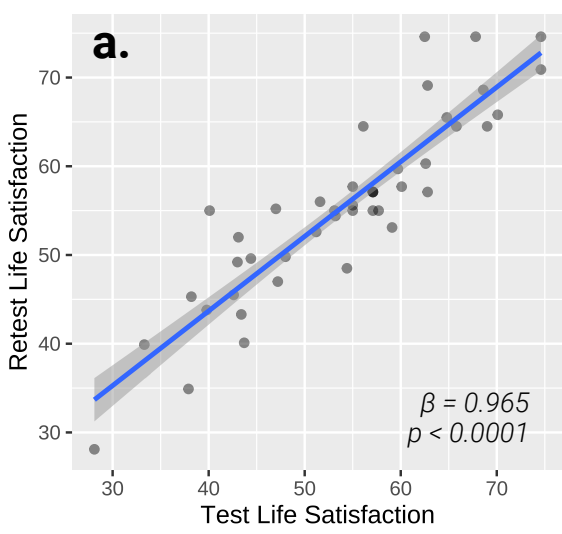

b.

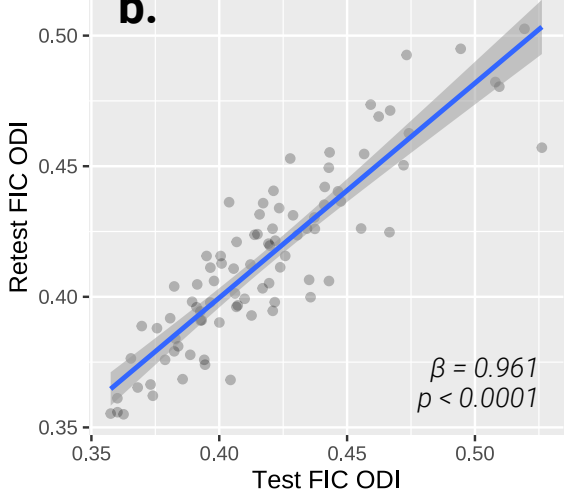

Cortical Microstructure Reliability

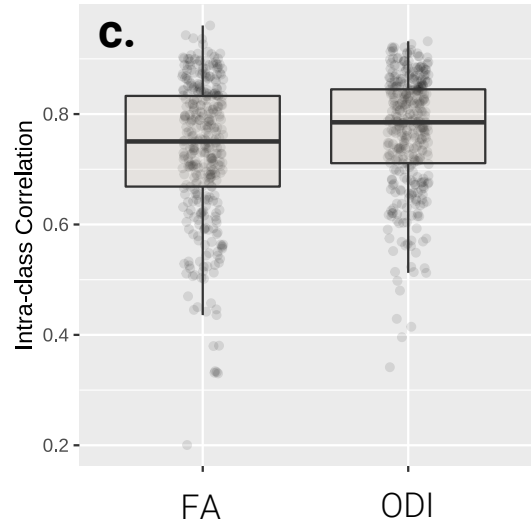

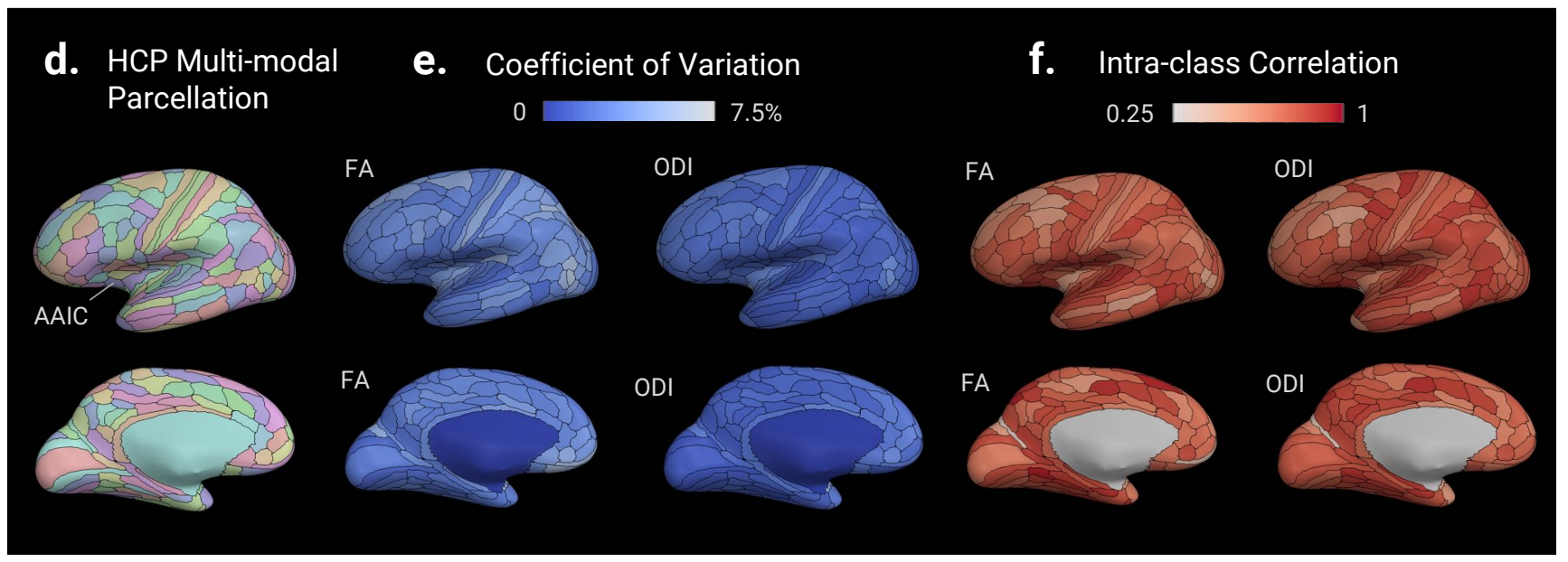

Fig. 3 Plots showing the results of our analysis of test-retest data to establish the reliability of our imaging metrics and reproducibility of our outcomes. The first row of panels shows a direct comparison between life satisfaction (a) and frontoinsular cortex (FIC) orientation dispersion (b) obtained from the same individual scanned in two different visits, where each dot represents a pair of measurements from a individual. The statistical results report the correlation $\beta$ and p-value. The next panel (c) shows results from an extended reliability analysis that includes all cortical regions from the HCP multi-modal parcellation, where each dot represents the intra-class correlation of a single cortical parcel. These results are plotted on an group-averaged inflated cortical surface in the next row, showing the HCP multi-modal parcellation (d), and per-parcel reliability scores for the coefficient of variation (e) and intra-class correaltion (f). The intraclass correlation measures the inter-scan variability relative to inter-subject variability (higher values are more reliable), and the coefficient of variation is the percentage variation across multiple scans (smaller is more reliable). Overall, the results demonstrate high agreement of frontoinsular metrics between the scanning sessions, as well as generally high reliability of cortical microstructure parameters, with slightly better performance in orientation dispersion than fractional anisotropy.

\section{Discussion}

The results reported here demonstrate a robust linkage bemicrostructural features of cortical gray matter in young adulsos hood. Specifically, we show these findings are specific to frontoinsular cortex, and not other cortical and subcortical brain areas; furthermore, our observed microstructural associations are independent of other behavioral and socioeconomic factors that otherwise relate to life satisfaction itself. 610 We obtained very similar results from anterior agranular insular cortex (AAIC) which was delineated by Freesurfer and substantially overlaps with the manually defined frontoinsular cortex based on expert knowledge of the area. Further evidence for the robustness of these findings comes from a set of 40 individuals in which both the life satisfaction measure and microstructural brain imaging were repeated at varying intervals after the initial observations had been made. Both the life satisfaction measures and their relationships with ODI and FA were well maintained upon retesting and were independent of the length of the interval between the initial test and the repeat. We also showed that these cortical microstructural metrics have a moderate degree of lateralization and heritability.

Life satisfaction is understood to be multi-faceted in nature (Peterson et al. 2005), and in our examination of demographic variables we also found a diverse set of associations among behavioral and socioeconomic factors. In 

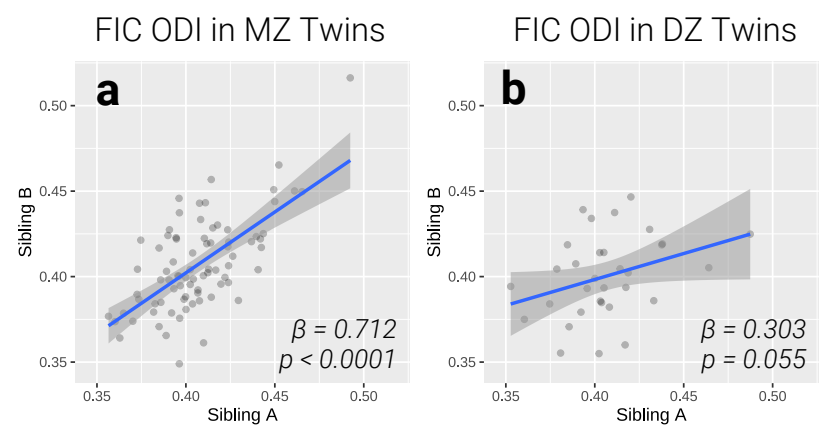

FIC ODI in Non-Twins
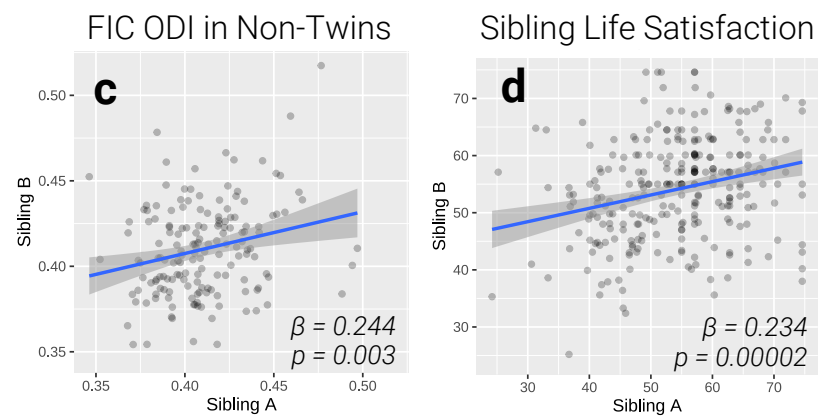

Fig. 4 Plots showing the results of our analysis of heritability in the sibling cohort. The siblings pairs were subdivided into monozygotic (MZ) twins (a), dizygotic (DZ) twins (b), and non-twin siblings (c), and the frontoinsular cortex (FIC) microstructure parameters corresponding to each twin pair are plotted. The fourth panel (d) shows the heritability of life satisfaction among all siblings. The shown statistical results report the paired correlation $\beta$ and associated $\mathrm{p}$-value between the siblings. Overall, the data show high heritability of microstructure ${ }_{635}$ in MZ twins and lower heritability in non-twin siblings, with intermediate and trend-level heritability in DZ twins. Life satisfaction showed significant heritability in all siblings, but with a lower effect size than microstructure.

particular that life satisfaction reflects socioeconomic variation in income, relationship status, education, and employment, which are in line with previous findings by Melin et al. (2003), who found that education, being vocationally active, and having a steady partner were predictive of life satisfac- -645 tion. Beyond this, others have found relevant mediation effects with relationship status Zhu et al. (2018), loneliness and social support (Kong and You, 2013). We further found associations with other measures of subjective well-being, findings that support previous work showing the connection ${ }_{650}$ of life satisfaction to different orientations to happiness ( $\mathrm{Pe}-$ terson et al. 2005). In contrast, we found the microstructural association with life satisfaction to be independent of these same behavioral and socioeconomic factors. The two significant covariates explaining variance in microstructure were 655 thought problems (negative and self-destructive thoughts) and sex, with the latter having a stronger effect. However, these factors showed no direct relation to life satisfaction, and thus the results may reflect the diverse function of frontoinsular cortex, which partly contributes to life satisfac-660

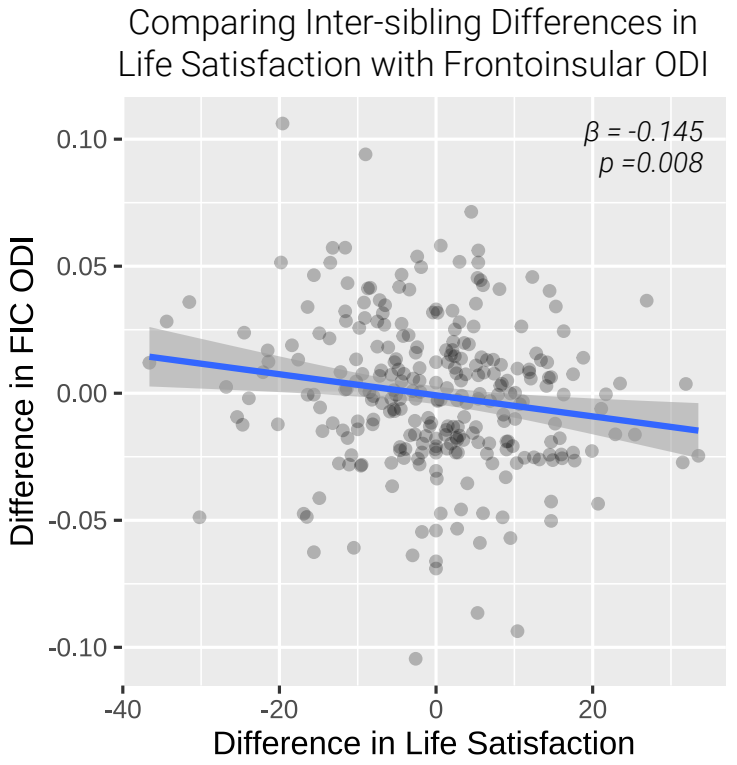

Fig. 5 A comparison of life satisfaction and microstructure in siblings. For each sibling pair, the inter-sibling differences in life satisfaction and microstructure were computed and plotted, where each dot represents a related pair of individuals. The data show an analogous pattern to our main outcome, where positive differences in life satisfaction statistically correspond to negative differences in microstructure, and vice-versa.

tion. These observed sex differences are notably divergent from previous work examining diffusion metrics in cortical gray matter (Schmitz et al., 2019); however, besides coming from a different population, there are methodological differences in the present work, wherein we used approaches for NODDI model fitting and surface-based cortical mapping which may provide more robust parameter maps.

Life satisfaction is often approached theoretically with the concept of a set-point, that is, there are regulatory system that maintain a constant level over time that is unperturbed by life events and changing circumstances (Fujita and Diener, 2005). Frontoinsular cortex is understood to be involved in processes of interoception, affective awareness, and error prediction, which are all critical to homeostatic regulation (Critchley, 2005, Craig, 2009). Homeostasis and set-point theory offer somewhat converging concepts, but there are open questions related to their temporal stability. Lykken and Tellegen (1996) conducted a large twin study and concluded that level of happiness is a strongly determined genetic trait (heritability $=0.44$ to 0.52 ), suggesting a stable set-point over life. More recently, the heritability of life satisfaction specifically has been measured in 10 separate twin studies (total $\mathrm{N}=44,450$ ). A meta-analysis of these studies found an average heritability index of 0.32 with a $95 \%$ confidence range between 0.29 and 0.35 (Bartels 2015). While these finding indicate a moderate genetic ba- 
sis for life satisfaction, thus supporting the set-point theory, they also imply a fair degree of lability over time, depen-715 dent on life circumstances. Longitudinal studies have shown that stability varies among different subsets of the subjects and tend to be less stable over time than biometric variables such as height, BMI and blood pressure (Fujita and Diener, 2005). We did not find any changes with age, butz20 given the limited age range of our cohort, a larger study is needed to determine how microstructural parameter retels (2015) found stability only for some individuals over time, and (Kubiszewski et al. 2020) found stability only in ${ }_{25}$ a high life satisfaction sub-cohort. In line with these findings, we observed moderate heritability of imaging-derived microstructure parameters, which suggests the possibility of some amount of shared genetic basis for frontoinsular characteristics and life satisfaction. Our comparison of inter-sibling differences showed a similar outcome to our main findings, which provides further support.

We may consider a number of neurobiological factors that explain our findings, and as our findings were localized to only frontoinsular cortex, we may focus on diffu $^{-735}$ sion MRI features of gray matter architecture. These effects were limited to differences in the orientation dispersion index, and by extension fractional anisotropy. While there are many factors that are known to affect fractional anisotropy, the biophysical and multi-compartment modeling approach ${ }^{70}$ of NODDI may allow for some of these to be differentiated. Specifically, this may reduce the influence of partial vol-

690 ume effects with cerebrospinal fluid and perivascular space effects (Sepehrband et al. 2019), and separate orientationdependent effects from neurite density. Theoretically, the ${ }^{75}$ orientation dispersion index reflects the heterogeneity in the distribution in the direction taken by neurites, so increased ODI may suggest differences in dendritic complexity (Broad et al. 2019), the presence of undulating fibers (Hagslätt et al. 2010), or perhaps, relative differences in distinct morpho- ${ }^{-70}$ logical cell types that make up frontoinsular cortex. This last point raises a question if these findings may be explained variation in von Economo Neurons (VENs) (Nimchinsky et al. 1999). VENs are large neurons with a distinct spindleshaped bipolar morphology, oriented radially and extend-755 ing over several cortical layers (Watson et al. 2006) (Allman et al. 2010) (Seeley et al., 2011), which presumably fractional anisotropy as their overall count increased. Previous work has found VENs to be preferentially depleted in ${ }^{760}$ fronto-temporal dementia (Seeley et al., 2006; Kim et al. 2012), and more prevalent in those with preserved cogni-

710 tion in extreme old age (Gefen et al., 2018). VENs are most abundant in frontoinsular and anterior cingulate cortices, and stere ological VEN counts have also consistently shown a higher 765 number in the right hemisphere (Allman et al. 2010), which is in accordance with the laterality observed in our findings of decreased ODI and increased FA with greater life satisfaction in frontoinsular cortex. However, VENs make up only a small portion of total cell count, so further validation work is necessary to determine the extent to which the distribution of VENs can be detected with MRI. Another factor to consider in our findings is that ODI may reflect changes in microglia density, a possibility which is supported by the observations of Yi et al. (2019), who used genetic tools in a rodent model to demonstrate a parametric association between microglia density and NODDI parameters.

This array of possible factors may also act in concert to produce the MR observable changes, and whether alone or together, they would likely produce alterations in the function of frontoinsular cortex. Previous work has found frontoinsular cortex to be a key component of regulatory processes in the brain (Allman et al. 2010, Nieuwenhuys, 2012), including interoceptive awareness (Craig, 2009), emotional awareness (Gu et al. 2013), salience processing (Seeley et al. 2007), which each play a part in homeostasis. Life satisfaction and well-being are the products of homeostatic mechanisms. The moderate degree of heritability of life satisfaction and well-being suggest that the homeostatic mechanisms underlying them are also heritable to some degree. The findings that the relationships between ODI and FA and life satisfaction are independent of the more transitory factors contributing to life satisfaction, such as relationships and employment, imply that there might be a significant connection between these measures of cortical microcircuitry and the homeostatic bases of life satisfaction. A relevant element of life satisfaction is that it is based on an individuals own judgements and expectations, and indeed, frontoinsular cortex and anterior insular are involved in such processing, as it serves as interceptive monitor of well-being (Craig. 2011), is involved in prediction error and error awareness (Ullsperger et al. 2010), and is associated with humor and sight gags (Watson et al. 2007). Furthermore, this may extend to a more general sense of well-being, as we also found a significant microstructural association with meaning and purpose, albeit with a small effect that was limited to only right frontoinsular cortex (and not found in AAIC). Conversely, insular cortex is also related to negative thinking, through its participation in the functional salience network (Lydon-Staley et al., 2019), and insular volume is inversely related to depression (Clark et al., 2010). The aspects of insular cortex relate to our findings, as negative intrusive thinking, or thought problems, was a significant covariate in explaining microstructural variation in frontoinsular cortex.

We designed our experiments to use high quality data and methods, but they are not without limitations. MRI itrself introduces a limit on the quality and specificity of our measurements. Imaging artifact was minimized to the degree possible, but it still can affect our results; for example, 
diffusion imaging of orbitofrontal cortex and frontal pole is affected greatly by susceptibility artifact, and even with our correction for geometric distortion, we may not collect suf-820 ficient signal to model these areas and rule out effects that may occur there. In contrast, we found artifact to be relatively low in frontoinsular cortex, and found deformable image registration to be as effective as the multi-modal surfacebased approach in this region. In fact, we found several results had a larger effect size when using the manually drawn ROI. Besides artifact, there are also limitations related to dif- 825 fusion MRI modeling. Tissue microstructure is necessarily more complex than depicted by the diffusion signal, so some simplifications are required to derive useful models and to modate limited resources for scanning. NODDI makes simplifying assumptions regarding the diffusivities of the intra-cellular, extra-cellular, and free-water compartments, 830 which potentially bias results if they are incorrect (Guerrero et al. 2019). However, we tried to address this by performtimized for gray matter; furthermore, we added DTI analysis for comparison, as it makes fewer modeling assump-835 tions. One interesting result was that NODDI systematically was more sensitive to our observed effects, as evidenced by greater effect sizes and smaller p-values, which may suggest that the modeling choices in NODDI are reducing the variance in microstructural parameters that are relevant to life satisfaction. Despite these limitations, there are ways to $^{840}$ possibly address these issues in later work. For example, ad-

795 vanced diffusion imaging sequences, such as q-space trajectory imaging (Westin et al. 2016) and diffusion-relaxometry (Gong et al. 2020), can potentially resolve differences between microscopic anisotropy and cell size; furthermore, $\mathrm{a}_{845}$ combination of MRI and microscopy can reveal and validate the precise relationship between cellular level features and those observable with MRI (Goubran et al. 2019). These approaches are not yet feasible at a scale comparable to the Human Connectome Project, but future studies employing these methods could be focused specifically on frontoinsu- ${ }^{850}$

805 lar cortex, given our results.

\section{Conclusion}

In conclusion, this study sheds new light on neurobiological factors that may underly subjective well-being in young adults. Using a large cohort of high-quality data from the $\mathrm{e}_{55}$ Human Connectome Project and state-of-the-art image modeling and analysis techniques, our experiments identified the orientation dispersion index (and by extension, fractional anisotropy) of frontoinsular cortex as a robust anatomicallyspecific diffusion MRI feature that is linked to life satisfaction, independent of other demographic, socioeconomic, and behavioral factors. We further validated our finding in a secondary test-retest dataset showing high reliability of our imaging metrics and reproducibility of our outcomes. Our results suggest a possible link with microscopic features of frontoinsular cortex, which may reflect cellular morphology and architecture; more broadly, they may implicate the integrity of the homeostatic processing performed by frontoinsular cortex as an important component of an individual's judgements of life satisfaction.

\section{Declarations}

Funding. This work was supported by National Institutes of Health (grant number P41EB015922) and made possible in part by grant number 2020-225670 from the Chan Zuckerberg Initiative DAF, an advised fund of Silicon Valley Community Foundation. Data were provided in part by the Human Connectome Project, WU-Minn Consortium (Principal Investigators: David Van Essen and Kamil Ugurbil; 1U54MH091657) funded by the 16 NIH Institutes and Centers that support the NIH Blueprint for Neuroscience Research; and by the McDonnell Center for Systems Neuroscience at Washington University.

Conflicts of interest/Competing interests. The authors have no conflict of interest to report.

Ethics approval. This project received approval from the University of Southern California Institutional Review Board and approval from the Human Connectome Project for Restricted Access from ConnectomeDB.

Consent to participant. Written informed consent was obtained from all individual participants as part of the conduct of the Human Connectome Project.

Availability of data and material. Data used in our study is available with permission from the Human Connectome Project ${ }^{2}$ Our data image analysis and visualization tools available online as part of the Quantitative Imaging Toolkit (QIT) 34

\section{References}

A. Abdellaoui, M. H. de Moor, L. M. Geels, J. H. Van Beek, G. Willemsen, and D. I. Boomsma. Thought problems from adolescence to adulthood: Measurement invariance and longitudinal heritability. Behavior Genetics, 42(1): 19-29, 2012.

T. M. Achenbach and L. Rescorla. Manual for the ASEBA adult forms \& profiles, 2003.

\footnotetext{
2 https://www.humanconnectome.org/study/ hcp-young-adult/data-releases

5 https://cabeen.io/qitwiki

4 https://resource.loni.usc.edu/resources/ downloads/
} 
D. C. Alexander, T. B. Dyrby, M. Nilsson, and H. Zhang. Imaging brain microstructure with diffusion mri: practicality and applications. NMR in Biomedicine, 32(4): e3841, 2019.

J. M. Allman, N. A. Tetreault, A. Y. Hakeem, K. F. Manaye,, 15 K. Semendeferi, J. M. Erwin, S. Park, V. Goubert, and P. R. Hof. The von Economo neurons in frontoinsular and anterior cingulate cortex in great apes and humans. Brain Structure and Function, 214(5-6):495-517, 2010.

B. B. Avants, C. L. Epstein, M. Grossman, and J. C. Gee.920 Symmetric diffeomorphic image registration with crosscorrelation: evaluating automated labeling of elderly and neurodegenerative brain. Medical Image Analysis, 12(1): 26-41, 2008.

M. Bartels. Genetics of wellbeing and its components satis-925 faction with life, happiness, and quality of life: A review and meta-analysis of heritability studies. Behavior genetics, 45(2):137-156, 2015.

P. J. Basser and D. K. Jones. Diffusion-tensor MRI: theory, experimental design and data analysis-a technical review.930 NMR in Biomedicine: An International Journal Devoted to the Development and Application of Magnetic Resonance In Vivo, 15(7-8):456-467, 2002.

M. Baxi, M. A. Di Biase, A. E. Lyall, S. Cetin-Karayumak, J. Seitz, L. Ning, N. Makris, D. Rosene, M. Kubicki, and ${ }_{935}$ Y. Rathi. Quantifying genetic and environmental influence on gray matter microstructure using diffusion mri. Cerebral Cortex, 2020.

R. J. Broad, M. C. Gabel, N. G. Dowell, D. J. Schwartzman, A. K. Seth, H. Zhang, D. C. Alexander, M. Cercig-940 nani, and P. N. Leigh. Neurite orientation and dispersion density imaging (noddi) detects cortical and corticospinal tract degeneration in als. Journal of Neurology, Neurosurgery \& Psychiatry, 90(4):404-411, 2019.

R. Cabeen, D. Laidlaw, and A. Toga. Quantitative Imaging ${ }_{945}$ Toolkit: Software for Interactive 3D Visualization, Data Exploration, and Computational Analysis of Neuroimaging Datasets. In Proc International Society for Magnetic Resonance in Medicine (ISMRM), volume 2018:2854, 2018.

R. Cabeen, F. Sepehrband, and A. Toga. Rapid and Accurate NODDI Parameter Estimation with the Spherical Mean Technique. In Proc International Society for Magnetic Resonance in Medicine (ISMRM), volume 2019:3363, 2019.

R. P. Cabeen, J. M. Allman, and A. W. Toga. THC exposure is reflected in the microstructure of the cerebral cortex and amygdala of young adults. Cerebral cortex, 2020.

B. Caron, D. Bullock, L. Kitchell, B. C. McPherson, D. A. Kellar, H. Cheng, S. D. Newman, N. L. Port, and ${ }_{960}$ F. Pestilli. Human white matter microstructure predicts elite sports participation. 2020.
D. L. Clark, N. N. Boutros, and M. F. Mendez. The brain and behavior: an introduction to behavioral neuroanatomy. Cambridge university press, 2010.

A. Craig. How do you feel-now? the anterior insula and human awareness. Nature Reviews Neuroscience, 10(1), 2009.

A. Craig. Significance of the insula for the evolution of human awareness of feelings from the body. Annals of the New York Academy of Sciences, 1225(1):72-82, 2011.

H. D. Critchley. Neural mechanisms of autonomic, affective, and cognitive integration. Journal of comparative neurology, 493(1):154-166, 2005.

E. Diener. Subjective well-being. In The science of wellbeing, pages 11-58. Springer, 2009.

E. Diener and M. E. Seligman. Beyond money: Toward an economy of well-being. Psychological science in the public interest, 5(1):1-31, 2004.

E. Diener, R. A. Emmons, R. J. Larsen, and S. Griffin. The satisfaction with life scale. Journal of Personality Assessment, 49(1):71-75, 1985. doi: 10.1207/ s15327752jpa4901 \_13. PMID: 16367493.

I. Dinov, J. Van Horn, K. Lozev, R. Magsipoc, P. Petrosyan, Z. Liu, A. MacKenzie-Graha, P. Eggert, D. S. Parker, and A. W. Toga. Efficient, distributed and interactive neuroimaging data analysis using the loni pipeline. Frontiers in Neuroinformatics, 3:22, 2009.

B. Fischl. Freesurfer. NeuroImage, 62(2):774-781, 2012.

F. Fujita and E. Diener. Life satisfaction set point: stability and change. Journal of personality and social psychology, 88(1):158, 2005.

H. Fukutomi, M. F. Glasser, H. Zhang, J. A. Autio, T. S. Coalson, T. Okada, K. Togashi, D. C. Van Essen, and T. Hayashi. Neurite imaging reveals microstructural variations in human cerebral cortical gray matter. NeuroImage, 182:488-499, 2018.

T. Gefen, S. T. Papastefan, A. Rezvanian, E. H. Bigio, S. Weintraub, E. Rogalski, M.-M. Mesulam, and C. Geula. Von economo neurons of the anterior cingulate across the lifespan and in alzheimer's disease. Cortex, 99: 69-77, 2018.

E. Genç, C. Fraenz, C. Schlüter, P. Friedrich, R. Hossiep, M. C. Voelkle, J. M. Ling, O. Güntürkün, and R. E. Jung. Diffusion markers of dendritic density and arborization in gray matter predict differences in intelligence. Nature communications, 9(1):1-11, 2018.

M. F. Glasser, S. N. Sotiropoulos, J. A. Wilson, T. S. Coalson, B. Fischl, J. L. Andersson, J. Xu, S. Jbabdi, M. Webster, J. R. Polimeni, et al. The minimal preprocessing pipelines for the human connectome project. NeuroImage, 80:105-124, 2013.

M. F. Glasser, T. S. Coalson, E. C. Robinson, C. D. Hacker, J. Harwell, E. Yacoub, K. Ugurbil, J. Andersson, C. F. Beckmann, M. Jenkinson, et al. A multi-modal parcel- 
lation of human cerebral cortex. Nature, 536(7615):171, 2016.

T. Gong, Q. Tong, H. He, Y. Sun, J. Zhong, and H. Zhang. Mte-noddi: Multi-te noddi for disentangling non- 2 to20 weighted signal fractions from compartment-specific $\mathrm{t} 2$ relaxation times. NeuroImage, page 116906, 2020.

X. Gu, P. R. Hof, K. J. Friston, and J. Fan. Anterior insular cortex and emotional awareness. Journal of Comparative $e_{30}$ Neurology, 521(15):3371-3388, 2013.

J. M. Guerrero, N. Adluru, B. B. Bendlin, H. H. Goldsmith, S. M. Schaefer, R. J. Davidson, S. R. Kecskemeti, H. Zhang, and A. L. Alexander. Optimizing the intrinsic parallel diffusivity in noddi: An extensive empirical eval ${ }_{+035}$ uation. PloS one, 14(9):e0217118, 2019.

H. Hagslätt, M. Nilsson, H. Hansson, J. Lätt, and D. van Westen. Diffusion mri on undulating versus straight axons: Reduced fractional anisotropy and increased apparent axonal diameter. 2010:1567, 2010.

M. Hlavac. stargazer: Latex code and ascii text for wellformatted regression and summary statistics tables. $U R L$ : http://CRAN. R-project. org/package= stargazer, 2013.

A. R. Hoy, C. G. Koay, S. R. Kecskemeti, and A. L. Alexander. Optimization of a free water elimination twot045 compartment model for diffusion tensor imaging. Neurolmage, 103:323-333, 2014.
F. Kong, S. Hu, X. Wang, Y. Song, and J. Liu. Neural correlates of the happy life: the amplitude of spontaneous low frequency fluctuations predicts subjective well-being. Neuroimage, 107:136-145, 2015b.

F. Kong, L. Liu, X. Wang, S. Hu, Y. Song, and J. Liu. Different neural pathways linking personality traits and eudaimonic well-being: a resting-state functional magnetic resonance imaging study. Cognitive, Affective, \& Behavioral Neuroscience, 15(2):299-309, 2015c.

F. Kong, X. Wang, S. Hu, and J. Liu. Neural correlates of psychological resilience and their relation to life satisfaction in a sample of healthy young adults. NeuroImage, 123:165-172, Dec. 2015d.

I. Kubiszewski, N. Zakariyya, R. Costanza, and D. Jarvis. Resilience of self-reported life satisfaction: A case study of who conforms to set-point theory in australia. PloS one, 15(8): $\mathrm{e} 0237161,2020$.

S. Kyeong, J. Kim, J. Kim, E. J. Kim, H. E. Kim, and J.-J. Kim. Differences in the modulation of functional connectivity by self-talk tasks between people with low and high life satisfaction. NeuroImage, page 116929, 2020.

G. J. Lewis, R. Kanai, G. Rees, and T. C. Bates. Neural correlates of the 'good life': eudaimonic well-being is associated with insular cortex volume. Social cognitive and affective neuroscience, 9(5):615-618, May 2014.

R. Li, X. Zhu, Z. Zheng, P. Wang, and J. Li. Subjective well-being is associated with the functional connectivity network of the dorsal anterior insula. Neuropsychologia, 141:107393, Apr. 2020.

D. Lydon-Staley, C. Kuehner, V. Zamoscik, S. Huffziger, P. Kirsch, and D. Bassett. Repetitive negative thinking in daily life and functional connectivity among default mode, fronto-parietal, and salience networks. Translational psychiatry, 9(1):1-12, 2019.

D. Lykken and A. Tellegen. Happiness is a stochastic phenomenon. Psychological science, 7(3):186-189, 1996.

L. Machado and A. Cantilino. Neural correlates of wellbeing scales: Preliminary data. The Australian and New Zealand journal of psychiatry, 51(9):946, Sept. 2017.

J. S. R. Mahmoud, R. Staten, L. A. Hall, and T. A. Lennie. The relationship among young adult college students' depression, anxiety, stress, demographics, life satisfaction, and coping styles. Issues in mental health nursing, 33(3): 149-156, 2012.

R. Melin, K. S. Fugl-Meyer, and A. R. Fugl-Meyer. Life satisfaction in 18-to 64-year-old swedes: in relation to education, employment situation, health and physical activity. Journal of rehabilitation medicine, 35(2):84-90, 2003.

A. Nazeri, C. Schifani, J. A. Anderson, S. H. Ameis, and A. N. Voineskos. In vivo imaging of gray matter microstructure in major psychiatric disorders: Opportunities for clinical translation. Biological Psychiatry: Cognitive Neuroscience and Neuroimaging, 2020. 
R. Nieuwenhuys. The insular cortex: a review. In Progress in Brain Research, volume 195, pages 123-163. Elsevier, 2012.

E. A. Nimchinsky, E. Gilissen, J. M. Allman, D. P. Perl ${ }_{125}$ J. M. Erwin, and P. R. Hof. A neuronal morphologic type unique to humans and great apes. Proceedings of the $\mathrm{Na}$ tional Academy of Sciences, 96(9):5268-5273, 1999.

V. Ourry, J. Gonneaud, B. Landeau, I. Moulinet, E. Touron, S. Dautricourt, G. Le Du, F. Mézenge, C. André, A. Be+130 janin, et al. Association of quality of life with structural, functional and molecular brain imaging in communitydwelling older adults. NeuroImage, page 117819, 2021.

W. M. Pauli, A. N. Nili, and J. M. Tyszka. A high-resolution probabilistic in vivo atlas of human subcortical brain nut135 clei. Scientific data, 5:180063, 2018.

W. Pavot and E. Diener. Review of the satisfaction with life scale. In Assessing well-being, pages 101-117. Springer, 2009.

C. Peterson, N. Park, and M. E. Seligman. Orientations t๑140 happiness and life satisfaction: The full life versus the empty life. Journal of happiness studies, 6(1):25-41, 2005.

R. M. Ryan and E. L. Deci. On happiness and human potentials: A review of research on hedonic and eudaimoni $\mathfrak{c}_{145}$ well-being. Annual review of psychology, 52(1):141-166, 2001.

C. D. Ryff, B. H. Singer, and G. Dienberg Love. Positive health: connecting well-being with biology. Philosophical Transactions of the Royal Society of London. Serie $\$ 150$ B: Biological Sciences, 359(1449):1383-1394, 2004.

J. M. Salsman, Z. Butt, P. A. Pilkonis, J. M. Cyranowski, N. Zill, H. C. Hendrie, M. J. Kupst, M. A. Kelly, R. K. Bode, S. W. Choi, et al. Emotion assessment using the nih toolbox. Neurology, 80(11 Supplement 3):S76-S86 2013.

J. M. Salsman, J.-S. Lai, H. C. Hendrie, Z. Butt, N. Zill, P. A. Pilkonis, C. Peterson, C. M. Stoney, P. Brouwers, and D. Cella. Assessing psychological well-being: selfreport instruments for the nih toolbox. Quality of Lif $e_{160}$ Research, 23(1):205-215, 2014.

1110 E. Samman. Psychological and subjective well-being: A proposal for internationally comparable indicators. $O x$ ford Development Studies, 35(4):459-486, 2007.

W. Sato, T. Kochiyama, S. Uono, Y. Kubota, R. Sawada,165 S. Yoshimura, and M. Toichi. The structural neural substrate of subjective happiness. Scientific reports, 5(1): 16891, Nov. 2015.

J. Schmitz, C. Fraenz, C. Schlüter, P. Friedrich, R. E. Jung, O. Güntürkün, E. Genç, and S. Ocklenburg. Hemispheri๔ $\varlimsup_{170}$ asymmetries in cortical gray matter microstructure identified by neurite orientation dispersion and density imaging. NeuroImage, 189:667-675, 2019.
W. W. Seeley, D. A. Carlin, J. M. Allman, M. N. Macedo, C. Bush, B. L. Miller, and S. J. DeArmond. Early frontotemporal dementia targets neurons unique to apes and humans. Annals of Neurology: Official Journal of the American Neurological Association and the Child Neurology Society, 60(6):660-667, 2006.

W. W. Seeley, V. Menon, A. F. Schatzberg, J. Keller, G. H. Glover, H. Kenna, A. L. Reiss, and M. D. Greicius. Dissociable intrinsic connectivity networks for salience processing and executive control. Journal of Neuroscience, 27(9):2349-2356, 2007.

W. W. Seeley, F. T. Merkle, S. E. Gaus, A. Craig, J. M. Allman, P. R. Hof, and C. Economo. Distinctive neurons of the anterior cingulate and frontoinsular cortex: a historical perspective. Cerebral Cortex, 22(2):245-250, 2011.

F. Sepehrband, R. P. Cabeen, J. Choupan, G. Barisano, M. Law, A. W. Toga, A. D. N. Initiative, et al. Perivascular space fluid contributes to diffusion tensor imaging changes in white matter. NeuroImage, 197:243-254, 2019.

D. C. Shin and D. M. Johnson. Avowed happiness as an overall assessment of the quality of life. Social indicators research, 5(1-4):475-492, 1978.

S. N. Sotiropoulos, S. Jbabdi, J. Xu, J. L. Andersson, S. Moeller, E. J. Auerbach, M. F. Glasser, M. Hernandez, G. Sapiro, M. Jenkinson, et al. Advances in diffusion MRI acquisition and processing in the Human Connectome Project. NeuroImage, 80:125-143, 2013.

J. R. Tanzer and L. Weyandt. Imaging happiness: Meta analysis and review. Journal of Happiness Studies, pages 142, 2019.

M. Torso, M. Bozzali, G. Zamboni, M. Jenkinson, S. A. Chance, and A. D. N. Initiative. Detection of alzheimer's disease using cortical diffusion tensor imaging. Human Brain Mapping.

J. M. Tyszka and W. M. Pauli. In vivo delineation of subdivisions of the human amygdaloid complex in a highresolution group template. Human Brain Mapping, 37 (11):3979-3998, 2016.

M. Ullsperger, H. A. Harsay, J. R. Wessel, and K. R. Ridderinkhof. Conscious perception of errors and its relation to the anterior insula. Brain Structure and Function, 214 (5):629-643, 2010.

D. C. Van Essen, S. M. Smith, D. M. Barch, T. E. Behrens, E. Yacoub, K. Ugurbil, W.-M. H. Consortium, et al. The WU-Minn human connectome project: an overview. Neurolmage, 80:62-79, 2013.

D. Van't Ent, A. den Braber, B. M. L. Baselmans, R. M. Brouwer, C. V. Dolan, H. E. Hulshoff Pol, E. J. C. de Geus, and M. Bartels. Associations between subjective well-being and subcortical brain volumes. Scientific reports, 7(1):6957, July 2017. 
K. K. Watson, T. K. Jones, and J. M. Allman. Dendritic architecture of the von Economo neurons. Neuroscience, 141(3):1107-1112, 2006.

K. K. Watson, B. J. Matthews, and J. M. Allman. Brain activation during sight gags and language-dependent humor. Cerebral cortex, 17(2):314-324, Feb. 2007.

C.-F. Westin, H. Knutsson, O. Pasternak, F. Szczepankiewicz, E. Özarslan, D. van Westen, C. Mattisson, M. Bogren, L. J. O'Donnell, M. Kubicki, et al. Q-space trajectory imaging for multidimensional diffusion mri of the human brain. Neuroimage, 135: 345-362, 2016

H. Wickham. The tidyverse. R package ver. 1.1, 1, 2017.

S. Y. Yi, B. R. Barnett, M. Torres-Velázquez, Y. Zhang, S. A. Hurley, P. A. Rowley, D. Hernando, and J.-P. J. Yu. Detecting microglial density with quantitative multicompartment diffusion mri. Frontiers in neuroscience, 13: 81, 2019.

H. Zhang, P. A. Yushkevich, D. C. Alexander, and J. C. Gee. Deformable registration of diffusion tensor $\mathrm{mr}$ images with explicit orientation optimization. Medical image analysis, 10(5):764-785, 2006.

H. Zhang, B. B. Avants, P. A. Yushkevich, J. H. Woo, S. Wang, L. F. McCluskey, L. B. Elman, E. R. Melhem, and J. C. Gee. High-dimensional spatial normalization of diffusion tensor images improves the detection of white matter differences: an example study using amyotrophic lateral sclerosis. IEEE transactions on medical imaging, 26(11):1585-1597, 2007.

H. Zhang, T. Schneider, C. A. Wheeler-Kingshott, and D. C. Alexander. Noddi: practical in vivo neurite orientation dispersion and density imaging of the human brain. NeuroImage, 61(4):1000-1016, 2012.

S. Zhang, H. Peng, R. J. Dawe, and K. Arfanakis. Enhanced ICBM diffusion tensor template of the human brain. $\mathrm{Neu}$ roImage, 54(2):974-984, 2011.

X. Zhu, K. Wang, L. Chen, A. Cao, Q. Chen, J. Li, and J. Qiu. Together Means More Happiness: Relationship Status Moderates the Association between Brain Structure and Life Satisfaction. Neuroscience, 384:406-416, Aug. 2018. 